\title{
Anatomic proportions of alveolar bone-A Radiographic Study Using Cone Beam Computed Tomography.
}

López-Jarana P, Díaz-Castro C, Falcão-Pereira A, Falcão-Pereira C, Herrero-Climent M. Porto dental Institute by Falcão.
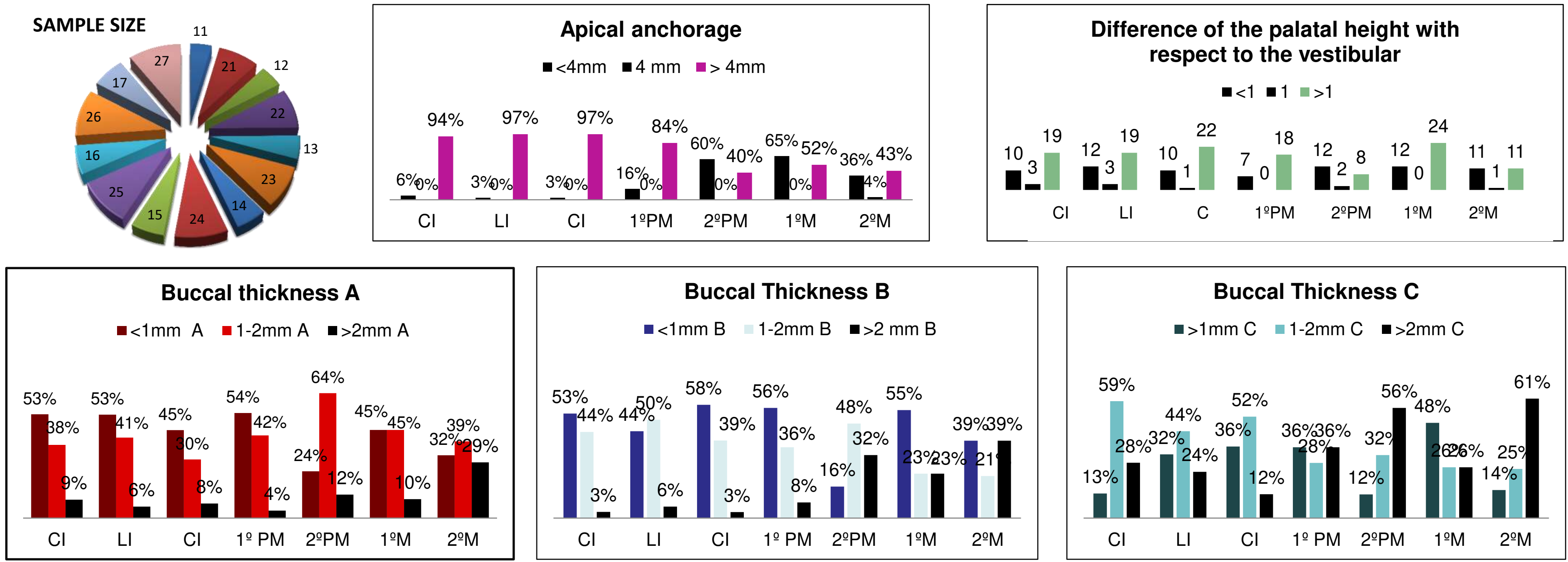

\section{- Introduction:}

Currently, the correct study of the alveolar bone morphology crucial and important factor in immediate implants. Since the introduction of the cone beam tomography (CBCT) it has developed into an essential diagnosis exam for adequate treatment planning, while also being a useful complementary exam due to the amount of data that a three-dimensional image provides to the dental clinician.

- Objective: to examine CBCT images by measuring four bone morphologic characteristics around maxillary teeth.

\section{- Materials and methods:}

The present retrospective study included CBCTs (Planmeca ProMax 3D) obtained using a spiral technique with $0,2 \mathrm{~mm}$ thickness $(200 \mathrm{~mm}$ field of view (FOV), $90 \mathrm{kV}, 10 \mathrm{mAs}, 1 \mathrm{~mm}$ pass) from patients referred to the department Of Periodontology of the Dental School of Seville (Spain) from January to December 2014. The size of the sample are 49 CBCT, with 208 teeth, 19 men and 30 women with an age range of 40,3 years (22-71).

Radiographic image analysis or CBCT evaluation: Three calibrated examiners made distinct measurements of the bone relative to the tooth in question.. Data was reconstructed with an DICOM image.

\section{- Parameters:}

1. The thickness of the facial bone wall was measured in a sagittal slice, perpendicular to the long axis of the root at several heights:

A: thickness at the coronal part of the facial crest

B: thickness at $4 \mathrm{~mm}$ from the coronal facial crest

C: thickness at the apex from the apical constriction to facial wall

D: the palatal thickness perpendicular to the coronal crest, near to buccal point $A$.
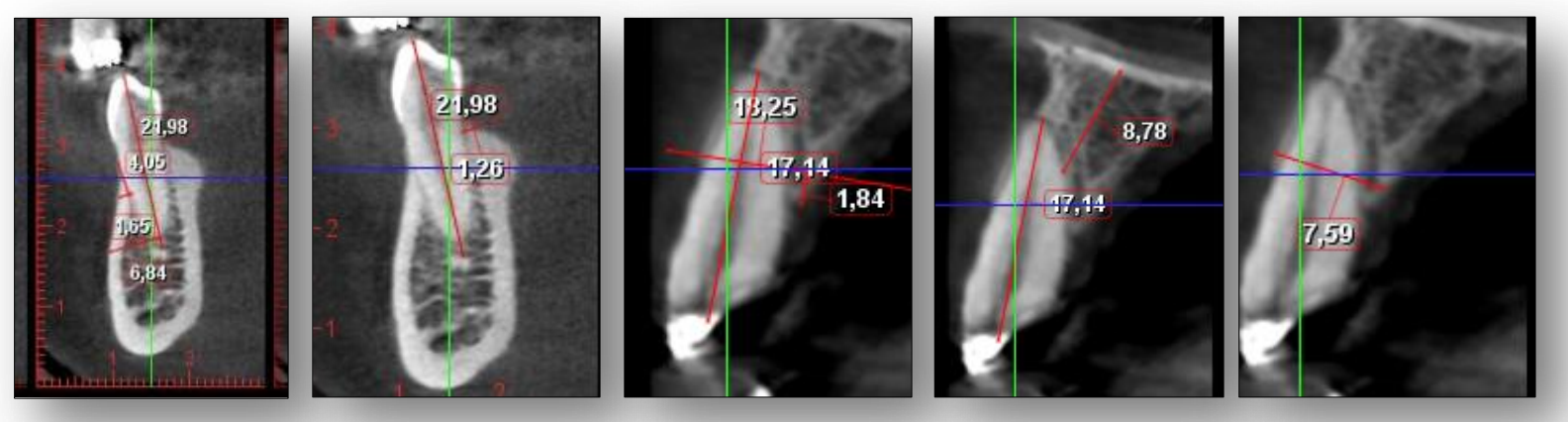

2. Apical anchorage: a line from the most coronal point where implant bone preparation would start up to an anatomical (bone partitions, nerve paths ...) that is perpendicular to the bisector of the remaining alveolus.

3. Alveolus width: a line from the vestibular crest to the palatal crest in the coronal portion, closest to the LAC.

4. Differences from the palatal height with respect to the vestibular: once traced the axial axis of the tooth, a perpendicular line that passes through the vestibular crest is drawn. The distance between the perpendicular line and the ridge on the palatine is measured by drawing a line that is in turn, parallel to the axial axis of the tooth.

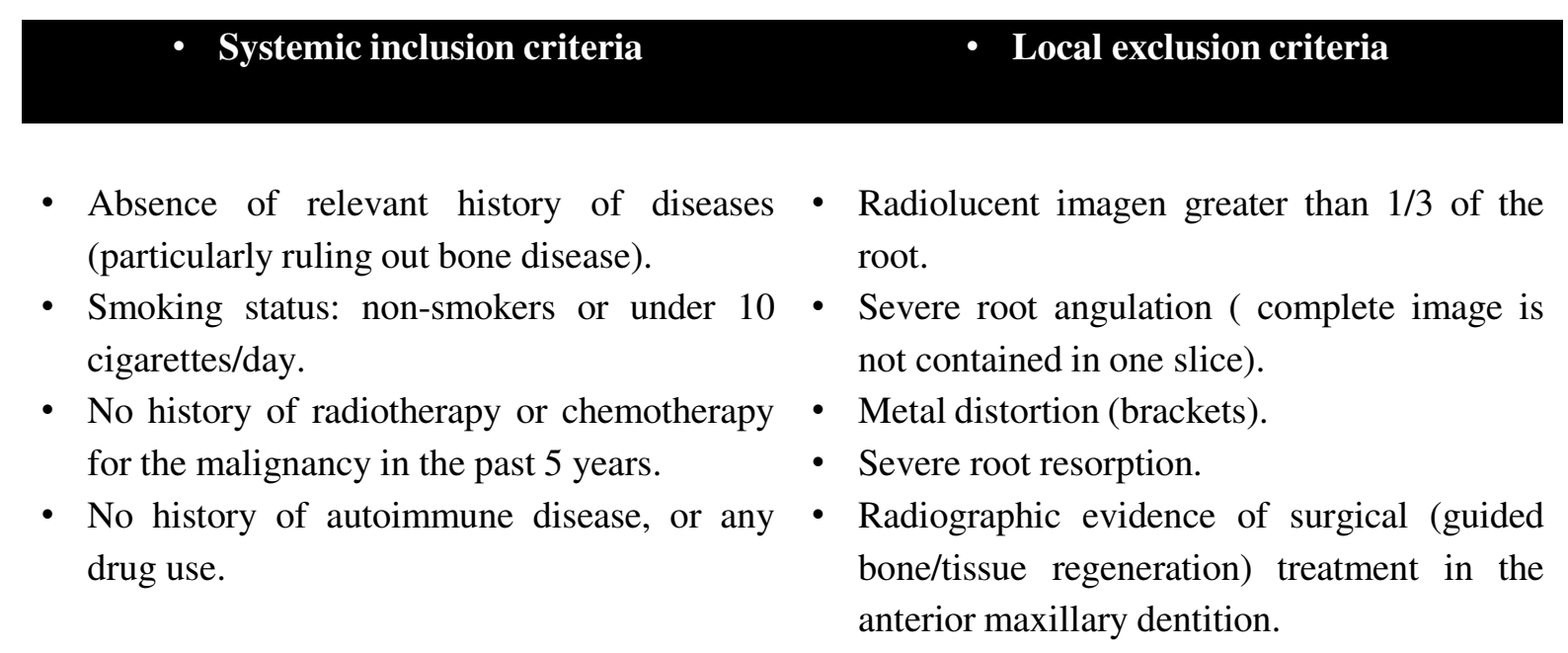

\begin{tabular}{cccccc}
\hline Palatal thickness & Valid & Mean & SD & Minimum & Maximum \\
& & & & & \\
\hline Incisive & 66 & 1,7 & 0,95 & 0,6 & 5,2 \\
Canines & 33 & 2,2 & 1,75 & 0,1 & 8,6 \\
Premolars & 50 & 1,6 & 0,89 & 0,4 & 4,0 \\
Molars & 59 & 1,9 & 1,51 & 0,0 & 8,1 \\
\hline & & & & & \\
\hline Alveolar width & Valid & Mean & SD & Minimum & Maximum \\
& & & & & \\
\hline Incisive & 66 & 7,8 & 1,44 & 5,7 & 11,9 \\
Canines & 33 & 8,8 & 1,35 & 5,3 & 11,6 \\
Premolar & 50 & 9,5 & 1,92 & 6,8 & 16,9 \\
Molars & 59 & 11,9 & 1,88 & 7,6 & 16,4 \\
\hline
\end{tabular}

- Discussion:

In the upper jaw, the $89,4 \%$ of incisors, the $93.94 \%$ of canines, $78 \%$ of premolars and $70,5 \%$ of molars had a thickness inferior to the ideal 2 $\mathrm{mm}$ of buccal alveolar process as in other studies like Huynh-Ba 2010. Recent studies of the alveolar width by Zang 2015, found similar results for central incisors, lateral and canines $(9,55-8,30-9,62)$. Our mean values are between 7,8-11,9 from incisor to molars. In the Nishihara et al 2017 study they find mean values of $9,4 \pm 1,1 \mathrm{~mm}$ for $1^{\circ}$ $\mathrm{PM}$ and $11,0 \pm 0,6 \mathrm{~mm}$ for $2^{\circ} \mathrm{PM}$. The anterior zone is the best place to insert a immediate implant in terms of apical anchorage, while the mean values of buccal thickness advice against these locations. The difference between vestibular and palatal crest tends to increase in premolars and molars sites, this means, from a clinical perspective, that the implant can be exposed during healing if it was not submerged at least $1-2 \mathrm{~mm}$. The thickness of the palatal bone in mean values are higher than in vestibular, so this seems to be an ideal zone to achieve stability and insertion torque when placing an immediate implant.

\section{- Conclusions:}

The alveolar bone thickness of the buccal plate was observed as thinner than $2 \mathrm{~mm}$ in the upper jaw, but only in canines for the palatal plate. Apical anchorage for immediate implant could be possible in anterior teeth. The mean values of alveolar width increase from anterior to posterior teeth. 